\title{
Spectral Modification by Diffraction and Scattering
}

\author{
M. Taghi Tavassoly, ${ }^{1}$ Masoomeh Dashtdar, ${ }^{2}$ and Mohammad Amiri ${ }^{3}$ \\ ${ }^{1}$ Physics Department, University of Tehran, 14395-547 Tehran, Iran \\ ${ }^{2}$ Department of Physics, Shahid Beheshti University, Evin, 1983963113 Tehran, Iran \\ ${ }^{3}$ Physics Department, Bu-Ali Sina University, 65178 Hamedan, Iran \\ Correspondence should be addressed to M. Taghi Tavassoly, tavasoli@iasbs.ac.ir \\ Received 1 December 2009; Accepted 2 March 2010 \\ Academic Editor: Oleg V. Angelsky
}

Copyright ( $\odot 2010$ M. Taghi Tavassoly et al. This is an open access article distributed under the Creative Commons Attribution License, which permits unrestricted use, distribution, and reproduction in any medium, provided the original work is properly cited.

Different effects induce spectral changes, for example, correlation of source fluctuations, propagation of light, random changes in optical properties of a medium, diffraction and scattering from objects, and rough interfaces. We review the spectral changes that occur as a result of light diffraction from phase steps, and particularly we discuss the spectral changes in the neighborhood of phase singularities. We also review the redshift and blueshift in the spectra of the lights coherently and diffusely scattered from rough interfaces. In addition, we study the effects of roughness and incident angle on the spectral profiles of scattered lights in reflection and transmission modes.

\section{Introduction}

After being realized in 1986 [1] that the spectrum of radiation may change on propagation, a considerable volume of works has been carried out dealing with spectral changes induced by the correlated fluctuations in sources and changes in coherence properties of radiations [2, 3]. Since the spatial coherence width of a light beam increases with the distance from the source, any light beam is coherent to some extent that depends on the source size and the distance from the source. Thus, as a coherent or partially coherent polychromatic beam of light passes through a medium that imposes different phases on different parts of the beam, the beam scatters from these parts and interference of the scattered lights leads to spectral changes. In this paper we study the spectral changes that occur as a result of light diffraction from phase steps and light scattering from rough interfaces in reflection and transmission modes.

\section{Modified Spectrum of Diffracted Light}

When a fully or partially coherent beam of light experiences a sharp change in amplitude or in phase, redistribution of intensity occurs in space that is called diffraction. A sharp change in amplitude occurs as the beam passage is partly obstructed by an opaque object. A sharp change in phase happens as a beam of light is reflected from a step, Figure 1(a), or passes through a transparent plate immersed in a liquid or gas where a sharp change in refractive index is imposed at the plate edge, Figure 1(b). The lights diffracted from the neighborhood of the change-affected area interfere and lead to different spectra at different points in the diffraction field. The amount of spectral change varies from point to point, but it is remarkable and anomalous in the neighborhoods of phase singularities.

Gbur et al. [4] used the Fresnel-Kirchhoff integral and obtained the following expression:

$$
M(r, \lambda)=\frac{1}{\lambda}\left|\iint_{W} \frac{e^{i k R}}{R} d^{2} r^{\prime}\right|^{2}
$$

for spectral modifying function for a fully coherent spherical wave front passing through a lens, at points in the neighborhood of the geometrical focal point. In (1), $r$, $\lambda, k, d^{2} r^{\prime}$, and $R$ stand for the distance between the focal point and observation point, wavelength, wavenumber, surface element, and the distance between a point on the aperture and observation point. The above function leads to drastic spectral changes in the neighborhoods of the phase singularities on the symmetry axis. But intensities at these 


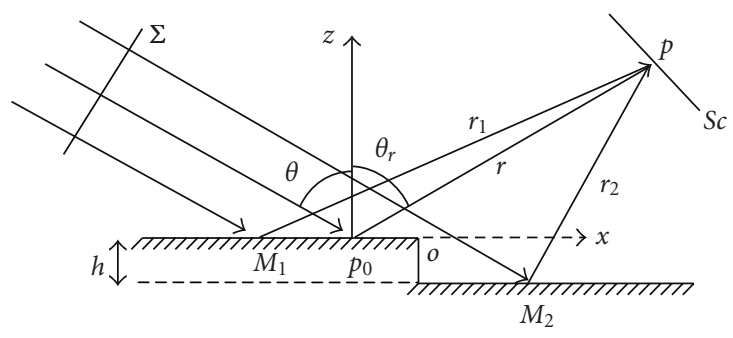

(a)

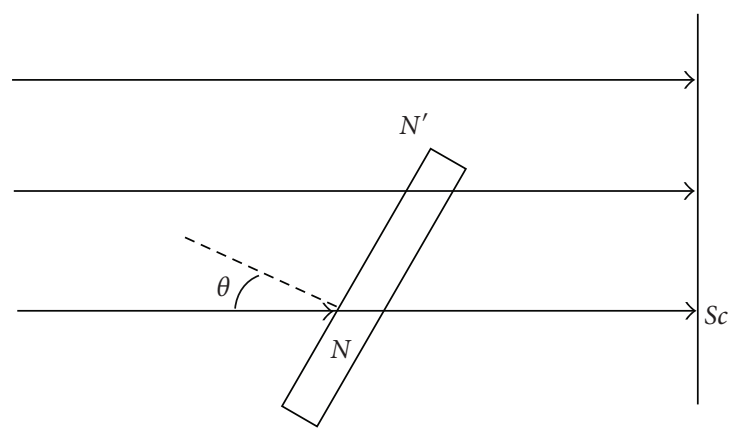

(b)

FIgURE 1: (a) The geometry of the Fresnel diffraction of light from a 1D phase step. By displacing point $p_{0}$ on the step, the point $p$ on the screen is shifted to satisfy equation $\theta_{r}=\theta$. Therefore, the complex amplitude varies as a function of $r$. (b) A phase step in transmission is formed at the edges of a transparent plate of refractive index $N$ that is immersed in a transparent gas or liquid of refractive index $N^{\prime}$.

parts are so low that challenges the experimental studies [5]. However, the diffracted intensities are quite appreciable at points corresponding to the neighborhoods of the phase singularities that are formed by the phase steps. In the following we describe these singularities with more details.

In Figure 1(a), the plane wave $\sum$ is incident to a $1 \mathrm{D}$ step of height $h$ at incident angle $\theta$. Using the FresnelKirchhoff integral, the diffracted complex amplitude at point $p$, the intersection of the screen and the ray that is specularly reflected from the step, can be given as $[6,7]$

$$
\begin{aligned}
U(r, \lambda)=\sqrt{\frac{-i}{\lambda}} A(\lambda) \frac{e^{i k r}}{\sqrt{r}} & {\left[\int_{-\infty}^{x_{0}} e^{i k x^{2} / 2 r} d x\right.} \\
& \left.+e^{i 2 k h \cos \theta} \int_{x_{0}}^{\infty} e^{i k x^{2} / 2 r} d x\right],
\end{aligned}
$$

where $A(\lambda)$ and $x_{0}$ stand for the amplitude of the incident light and the distance from $p_{0}$ to the step edge, $p_{0} o$, respectively. Using the abbreviations

$$
\frac{k x^{2}}{r}=\pi v^{2}, \quad 2 k h \cos \theta=\phi, \quad \frac{e^{i k r}}{\sqrt{r}}=u_{0},
$$

the complex amplitude at point $p$, for $p_{0}$ on the left side of the step edge, can be given by

$$
U_{L}(r, \lambda)=K^{\prime} A(\lambda)\left[\int_{-\infty}^{v_{L}} e^{i \pi v^{2} / 2} d v+e^{i \phi} \int_{v_{L}}^{\infty} e^{i \pi v^{2} / 2} d v\right]
$$

and for $p_{0}$ on the right side of the step edge by

$$
U_{R}(r, \lambda)=K^{\prime} A(\lambda)\left[\int_{-\infty}^{-v_{R}} e^{i \pi v^{2} / 2} d v+e^{i \phi} \int_{-v_{R}}^{\infty} e^{i \pi v^{2} / 2} d v\right]
$$

where $K^{\prime}=\sqrt{-i / 2} u_{0} ; v_{R}$ and $v_{L}$ are the Fresnel variables corresponding to the distance between the step edge and point $p_{0}$, for $p_{0}$ on the left and right sides of the step edge. The corresponding intensities are $[6,7]$

$$
\begin{gathered}
I_{L}(r, \lambda)=\frac{I_{0}(\lambda)}{r}\left[\cos ^{2}\left(\frac{\phi}{2}\right)+2\left(C_{L}^{2}+S_{L}^{2}\right) \sin ^{2}\left(\frac{\phi}{2}\right)\right. \\
\left.-\left(C_{L}-S_{L}\right) \sin \phi\right], \\
I_{R}(r, \lambda)=\frac{I_{0}(\lambda)}{r}\left[\cos ^{2}\left(\frac{\phi}{2}\right)+2\left(C_{R}^{2}+S_{R}^{2}\right) \sin ^{2}\left(\frac{\phi}{2}\right)\right. \\
\left.+\left(C_{R}-S_{R}\right) \sin \phi\right],
\end{gathered}
$$

where, $C_{L}, S_{L}$ and $C_{R}, S_{R}$ are the Fresnel cosine and sine integrals for the cases $p_{0}$ lying on the left and right sides of the step edge. These equations show that the diffracted light spectrum is modified, and the modifying functions are

$$
\begin{aligned}
M_{L}(r, \lambda)=[ & \cos ^{2}\left(\frac{\phi}{2}\right)+2\left(C_{L}^{2}+S_{L}^{2}\right) \sin ^{2}\left(\frac{\phi}{2}\right) \\
& \left.-\left(C_{L}-S_{L}\right) \sin \phi\right], \\
M_{R}(r, \lambda)=[ & \cos ^{2}\left(\frac{\phi}{2}\right)+2\left(C_{R}^{2}+S_{R}^{2}\right) \sin ^{2}\left(\frac{\phi}{2}\right) \\
& \left.+\left(C_{R}-S_{R}\right) \sin \phi\right] .
\end{aligned}
$$

Immersing a transparent plate of refractive index $N$ in a transparent gas or liquid of refractive index $N^{\prime}$, a phase step is formed in transmission. Calculating the amplitudes and intensities in the same manner that is done for a phase step in reflection leads to similar equations, but the phase change should be replaced by the following:

$$
\phi=k N^{\prime} h\left[\sqrt{n^{2}-\sin ^{2} \theta}-\cos \theta\right],
$$

where $n=N / N^{\prime}$.

At the exact edge, that is, for $v_{R}=v_{L}=0$ and

$$
\phi=(2 m+1) \pi, \quad m=0, \pm 1, \ldots,
$$




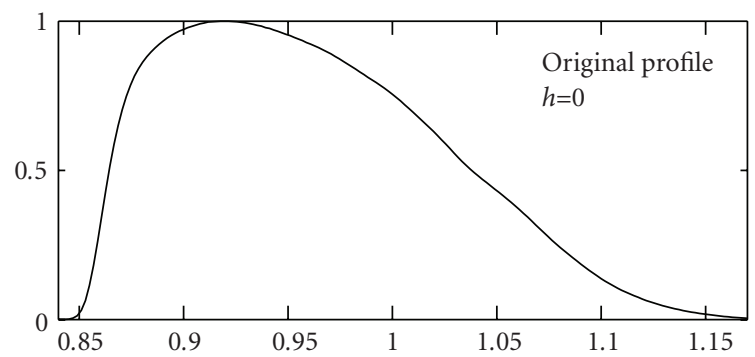

(a)

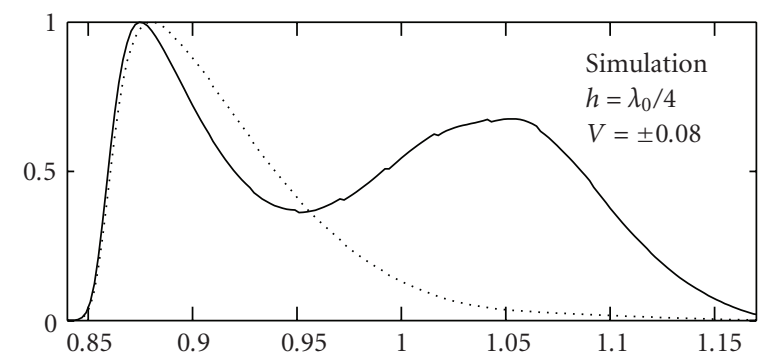

(b)

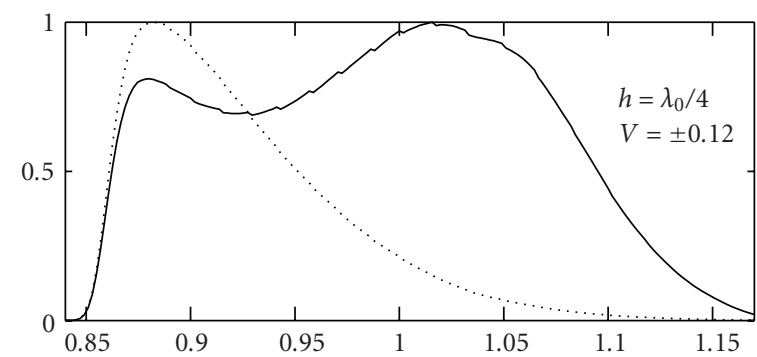

(c)

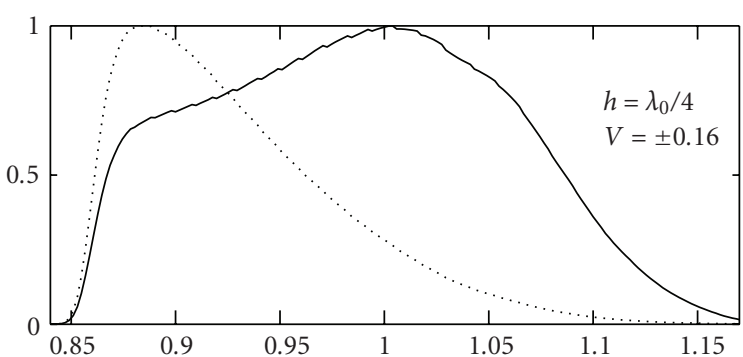

(d)

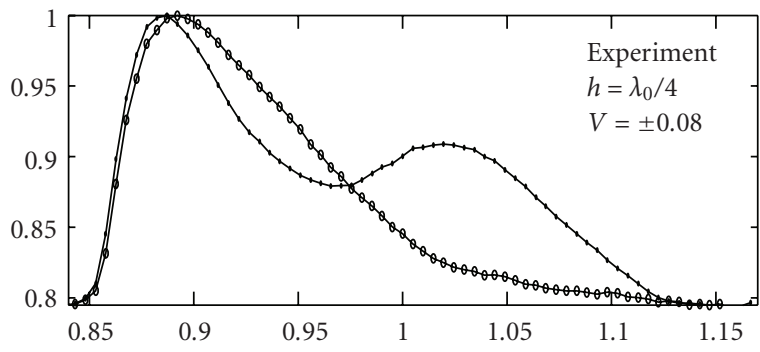

(b')

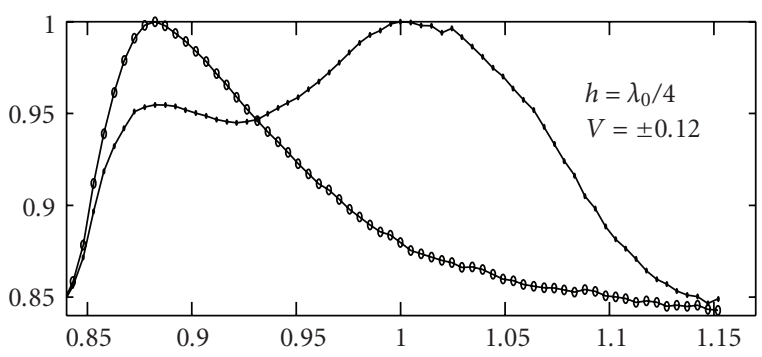

$\left(c^{\prime}\right)$

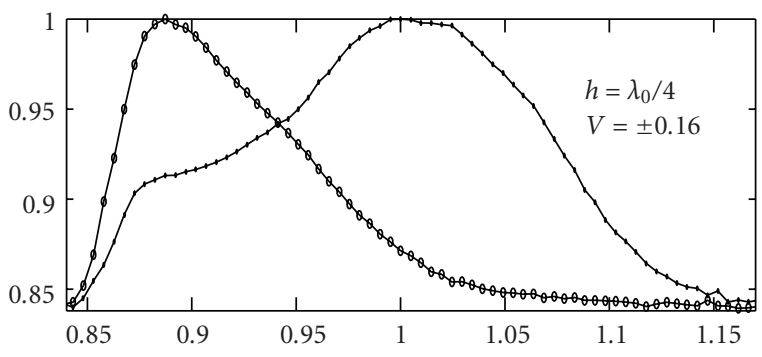

(d')

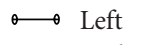

طight

FIgUre 2: (a) The spectral profile of the light incident to a phase step formed by the Michelson interferometer versus $\lambda / \lambda_{0}\left(\lambda_{0}=510 \mathrm{~nm}\right)$. The plots $\left(b^{\prime}\right),\left(c^{\prime}\right)$, and $\left(d^{\prime}\right)$ on the right are the experimental spectral profiles of the incident lights (a) diffracted from the step at points corresponding to different distances from the step edge. The plots (b), (c) and (d) on the left are the corresponding spectral profiles obtained by simulation.

we have phase singularity. Thus, by changing the step height or the incident angle $\theta$, one can create a phase singularity with the desired depth. For a phase singularity at $\lambda_{0}$, that is, for $4 \pi h \cos \theta / \lambda_{0}=(2 m+1) \pi$, the modifying function for wavelengths close to $\lambda_{0}$ at points near the step edge can be approximated by

$$
\begin{aligned}
& M_{L}(r, \lambda) \approx 2 C_{L}\left(C_{L}+\alpha\right), \\
& M_{R}(r, \lambda) \approx 2 C_{R}\left(C_{R}-\alpha\right),
\end{aligned}
$$




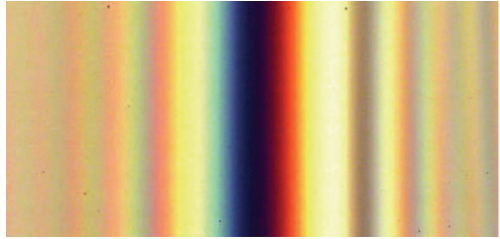

$\theta_{i}=26^{\circ}$

(a)

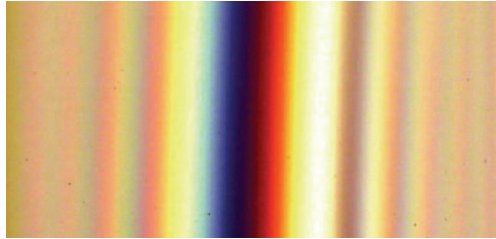

$\theta_{i}=35^{\circ}$

(b)

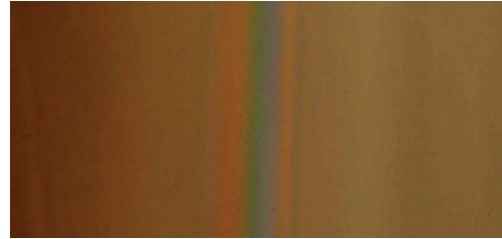

$\theta_{i}=58^{\circ}$

(c)

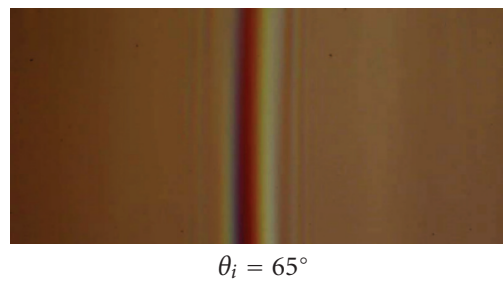

(d)

FIGURE 3: Diffraction patterns of spatially coherent white light diffracted from a $1 \mathrm{D}$ phase step of height $h=440 \mathrm{~nm}$ constructed by coating aluminum film on a glass substrate. The changes in colors occur by changing the light incident angle.

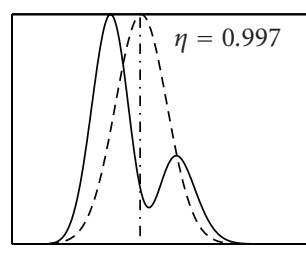

(b)

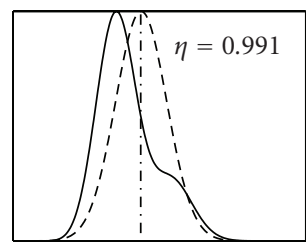

(a)

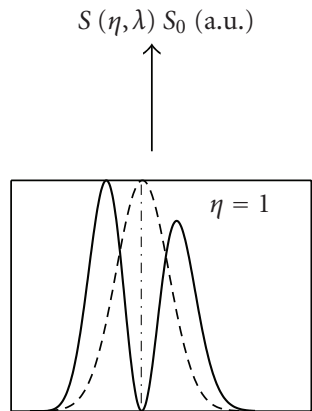

(c)

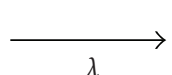

$\lambda$

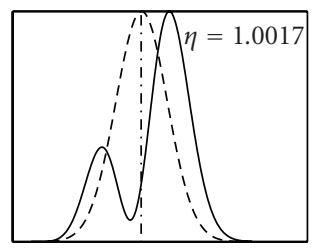

(d)

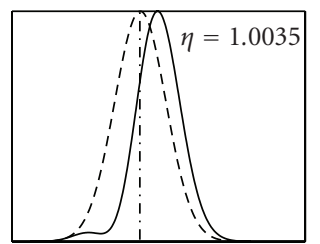

(e)

FIgure 4: Modified spectral profiles for TM component of the incident light in the neighborhood of Brewster angle $\theta_{B}$ for five different incident angles, $\eta=\theta / \theta_{B}$.

where $\alpha$ denotes the phase change that is obtained for replacing $\lambda_{0}$ by $\lambda$. For $\lambda>\lambda_{0}$, we have $\alpha<0$. That means that the spectra at points on the right side of the step edge are redshifted and at points on the left side are blueshifted, and the amount of modification increases by the distance from the step edge. However, the spectral modification becomes complicated by the further increase of the distance as a result of entering in Cornu spirals.

Phase steps can be formed in different ways. For example, if in the Michelson or Mach-Zehnder interferometer we block the alternative halves of the beams incident to the mirrors by two opaque plates, a 1D phase step is formed [8], and by displacing one of the mirrors, the height of the step is varied. Another simple way for fabricating $1 \mathrm{D}$ step is to coat a film on a substrate in the form of a step. In this case the phase $\phi$ can vary by changing the incident angle.
On the top of Figure 2 the spectrum of the light beam is plotted that was used to illuminate a $1 \mathrm{D}$ step formed in the Michelson interferometer. The other plots are the spectral profiles of the diffracted lights from the step at different distances from the step edge. The plots on the left side are the corresponding spectral profiles obtained by simulation. There are remarkable differences between the spectra corresponding to the same distances on the sides of the step edge.

In Figure 3 the diffraction patterns of white lights are shown, that diffracted from a step formed by coating aluminum on a substrate for different incident angles. The film thickness was $440 \mathrm{~nm}$.

Another interesting phase singularity happens at Brewster angle for TM component of incident light beam illuminating the surface of a dielectric at angles close to 


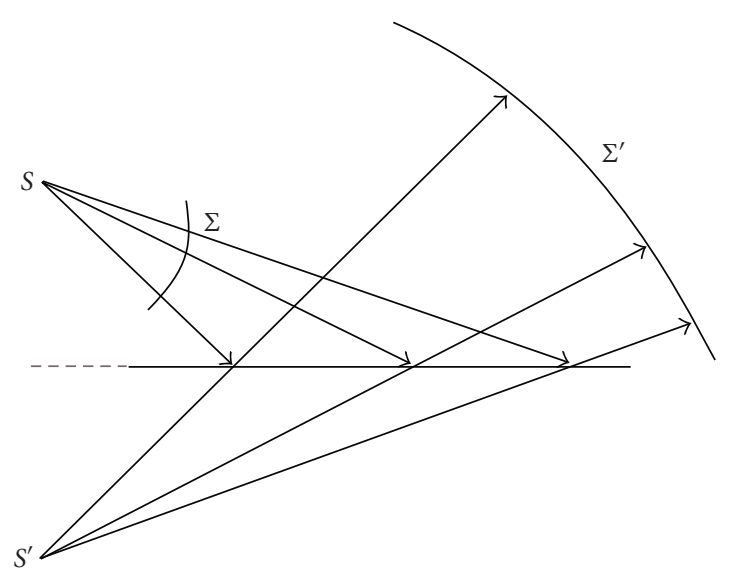

(a)

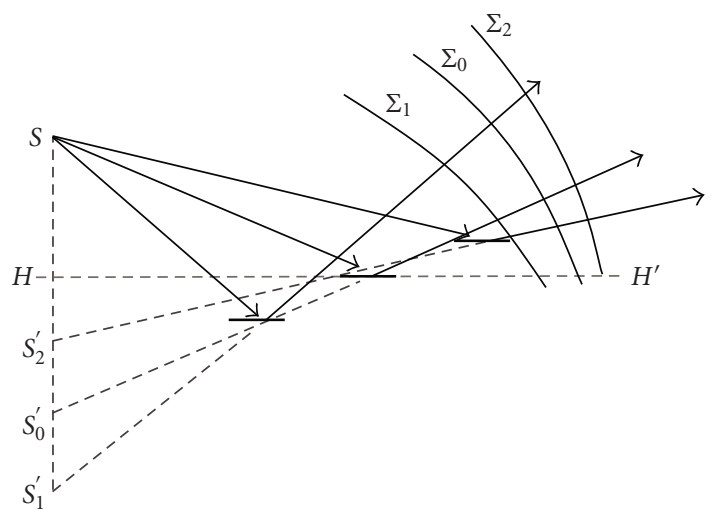

(b)

Figure 5: (a) The image of a point object is observed in a smooth surface because the reflected wave front is a continuous spherical surface. (b) The image is not observed in a very rough interface because the waves reflected from different parts of the rough interface do not form a continuous wave front.

the Brewster angle. For this component we have a step of height equivalent to a phase change of $\pi$ at Brewster angle. Using the Fresnel-Kirchhoff integral and applying the Fresnel equations for the amplitude reflection coefficients, the following modifying function is obtained [9]:

$$
M(\theta, \lambda)=\frac{K^{2} R}{\lambda}\left[C^{2}(\theta, \lambda)+S^{2}(\theta, \lambda)\right]
$$

where $K$ is the propagation factor, $R$ is the distance between the light incident point and observation point, $C(\theta, \lambda)$, and $S(\theta, \lambda)$ are the Fresnel sine and cosine integrals at incident angle $\theta$ that is close to the Brewster angle. In Figure 4 the modified spectral profiles in the neighborhood of the Brewster angle versus $\lambda$ are plotted for an incident light of Gaussian spectral profile. As the plots shown for angles less than Brewster angle $\theta_{B}, \eta<1\left(\eta=\theta / \theta_{B}\right)$, the spectrum is blueshifted, and for $\eta>1$ it is redshifted.

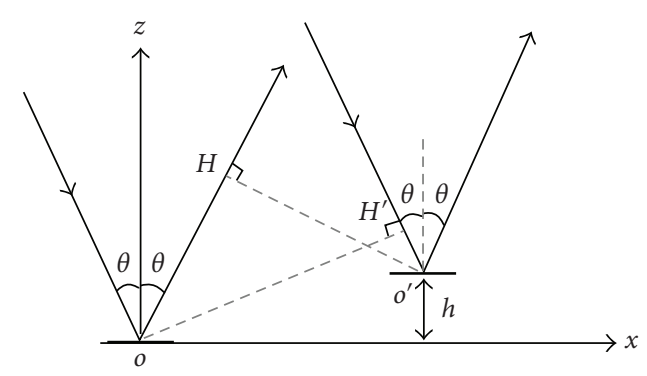

FIGURE 6: The optical path difference between the rays reflected from two parallel facets at arbitrary locations, $2 h \cos \theta$, reduces as incident angle increases.

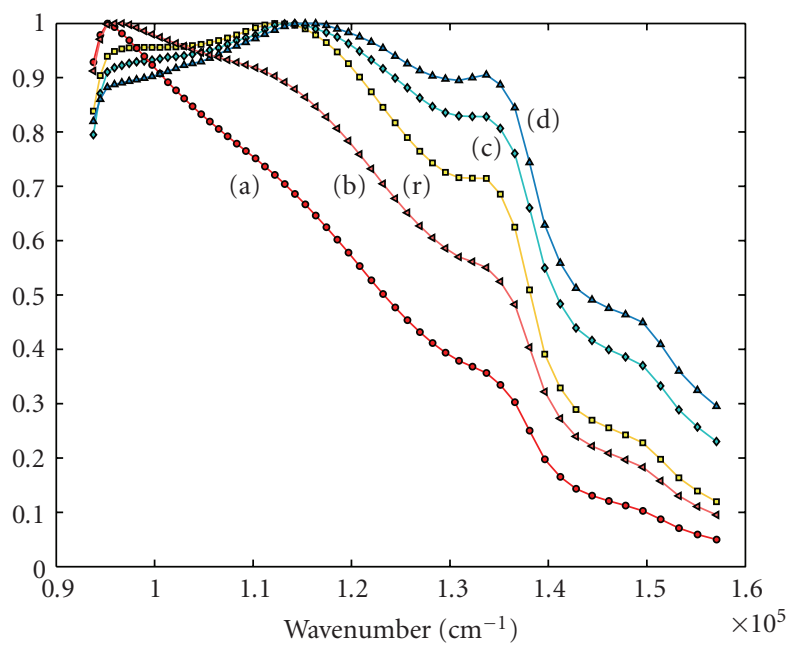

FIGURE 7: The measured spectral profiles of the incident light beam, (r), the coherently scattered lights, (a) and (b), and the diffusely scattered lights at scattering angle $\theta_{s}=1^{\circ},(\mathrm{c})$ and (d), from interfaces of roughness $\sigma=0.42 \mu \mathrm{m}$ and $\sigma=0.08 \mu \mathrm{m}$, respectively.

\section{Spectral Modification of the Light Scattered from Rough Interfaces}

When a spherical wave that is originated from a point object strikes a flat smooth surface, the reflected wave is coherent on a sphere centered at the image of the object, Figure 5(a). However, if a spherical wave is incident to a flat rough surface, we face different sections of waves reflected from a large number of facets at different heights that are not correlated. Therefore, the image is not observed, Figure 5(b). But as the object gets closer to the rough interface, in other words, for large incident angles, the waves diffracted from different facets become correlated, and for very large incident angles the spherical wave is restored and the image reappears. Thus, at very large incident angles rough surfaces look smooth. In fact, it is easy to see from Figure 6 that the optical path difference between the lights reflected from two facets at height difference $h$, at two arbitrary locations, is the following:

$$
\Delta=2 h \cos \theta .
$$


Thus, we conclude that when a coherent parallel beam of light is incident to a rough interface, depending on the roughness, incident angle, and light wavelength, there may be coherently scattered light in specular direction. However, by the increase of incident angle the spatial coherence property of light improves, and beyond a threshold incident angle we have partially coherent light in specular direction. Also we have diffusely scattered light in all directions. The partially coherent scattered lights interfere and lead to spectral modification, including redshift and blueshift. There are reports on spectral modification of light scattered from rough interfaces $[10,11]$. But recently rather detailed theoretical and experimental studies of the subject in reflection and transmission modes have been reported [12-15]. According to these works the amplitude of the coherently scattered monochromatic light from a randomly rough interface versus incident angle is the Fourier transform of the height distribution on the rough interface. In addition, for polychromatic incident light the coherently scattered light amplitude versus wave number is also the Fourier transform of the height distribution on the rough interface. Calculation of the coherently scattered light intensity leads to the following expression [13-15]:

$$
S\left(k, P_{n}, f\right)=S_{0}(k) \beta^{2}\left|\int_{-\infty}^{+\infty} P_{n}(h) \exp (-i k h f) d h\right|^{2},
$$

where $S_{0}(k), \beta$, and $P_{n}(h)$ denote the illuminating light intensity at wavenumber $k$, a dimensionless constant, and the probability density function of height distribution on the rough interface. Also, we should substitute

$$
f=2 \cos \theta_{i}
$$

in reflection mode and

$$
f=N_{1}\left(\sqrt{N^{2}-\sin ^{2} \theta_{i}}-\cos \theta_{i}\right)
$$

in transmission mode, where $\theta_{i}$ is the incident angle and $N=N_{2} / N_{1}$ where $N_{1}$ and $N_{2}$ are the refractive indices of the media surrounding the rough interface. As shown in (13) the coherently scattered light intensity depends on $k$, $f$, and $P_{n}(h)$. Thus, we have spectral modification with the following modifying function:

$$
M\left(k, P_{n}, f\right)=\beta^{2}\left|\int_{-\infty}^{+\infty} P_{n}(h) \exp (-i k h f) d h\right|^{2} .
$$

For an incident light of Gaussian spectral profile

$$
S_{0}(k)=s_{0} \beta^{2} \exp \left(-\frac{\left(k-k_{0}\right)^{2}}{2 \sigma_{k}^{2}}\right),
$$

the modified spectral profile becomes

$$
\begin{aligned}
S\left(k, p_{n}, f\right)= & S_{0} \exp \left(-k_{0}^{2} \sigma^{2} f^{2}\right) \\
& \times \exp \left[-\frac{\left(k-k_{0} /\left(1+2 \sigma_{k}^{2} \sigma^{2} f^{2}\right)\right)^{2}}{2 \sigma_{k}^{2} /\left(1+2 \sigma_{k}^{2} \sigma^{2} f^{2}\right)}\right],
\end{aligned}
$$

where $\sigma$ is the RMS of height distribution. We see that when $s_{0} \exp \left(-k_{0}^{2} \sigma^{2} f^{2}\right)$ is appreciable, we have the following redshift and spectral width shrinkage:

$$
\begin{gathered}
\delta k_{0}=\frac{k_{0}}{1+1 / 2 \sigma_{k}^{2} \sigma^{2} f^{2}}, \\
\sigma_{M}=\frac{\sigma_{k}}{\sqrt{1+2 \sigma_{k}^{2} \sigma^{2} f^{2}}} .
\end{gathered}
$$

According to (18) and (19) when $s_{0} \exp \left(-k_{0}^{2} \sigma^{2} f^{2}\right)$ is appreciable, the coherently scattered light spectrum is always redshifted with a shrinkage in spectral width. This result implies that in this situation the diffusely scattered light is blueshifted. Theoretical predictions and experimental results show that blueshift reduces with the increase of scattering angle. In addition, the spectral modification is significant near the threshold angle, the angle at which the coherently scattered light intensity becomes nonzero. In Figure 7 the spectral profiles of the incident beam, $(r)$, and the coherently scattered beams, (a) and (b), and the diffusely scattered lights at scattering angle $\theta_{s}=1^{\circ},(\mathrm{c})$ and (d), are plotted for interfaces having roughness $\sigma=0.42 \mu \mathrm{m}$ and $\sigma=0.08 \mu \mathrm{m}$, respectively.

\section{Conclusions}

(1) Spectral modification by diffraction of light from phase steps is a rich subject and can provide adequate ground for deeper studies of phase singularities.

(2) Polychromatic light diffraction from phase steps has applications in metrology, optical switching, and in dealing with transparent nonlinear materials.

(3) The scattering of polychromatic lights from rough interfaces gives better understanding of the optical field near the rough interfaces and it can provide valuable information about the properties of rough interfaces.

(4) The results obtained can be applied to transparent materials that impose irregular or random phases on lights propagating through them.

\section{References}

[1] M. Born and E. Wolf, Principles of Optics, 7th edition, 2002.

[2] E. Wolf and D. F. V. James, "Correlation-induced spectral changes," Reports on Progress in Physics, vol. 59, no. 6, pp. 771818, 1996.

[3] L. Mandel and E. Wolf, Optical Coherence and Quantum Optics, section 5.8., Cambridge University Press, Cambridge, UK, 1995.

[4] G. Gbur, T. D. Visser, and E. Wolf, "Anomalous behavior of spectra near phase singularities of focused waves," Physical Review Letters, vol. 88, no. 1, Article ID 013901, 4 pages, 2002.

[5] G. Popescu and A. Dogariu, "Spectral anomalies at wave-front dislocations," Physical Review Letters, vol. 88, no. 18, Article ID 0183902, 4 pages, 2002. 
[6] M. T. Tavassoly, M. Amiri, E. Karimi, and H. R. Khalesifard, "Spectral modification by line singularity in Fresnel diffraction from 1D phase step," Optics Communications, vol. 255, no. 1-3, pp. 23-34, 2005.

[7] M. Amiri and M. T. Tavassoly, "Fresnel diffraction from 1D and 2D phase steps in reflection and transmission modes," Optics Communications, vol. 272, no. 2, pp. 349-361, 2007.

[8] M. T. Tavassoly, M. Amiri, A. Darudi, R. Aalipour, A. Saber, and A. R. Moradi, "Optical diffractometry," Journal of the Optical Society of America A, vol. 26, no. 3, pp. 540-547, 2009.

[9] M. Amiri and M. T. Tavassoly, "Spectral anomalies near phase singularities in reflection at Brewster's angle and colored castastrophes," Optics Letters, vol. 33, no. 16, pp. 1863-1865, 2008.

[10] O. V. Angelsky, P. V. Polyanskii, and S. G. Hanson, "Singularoptical coloring of regularly scattered white light," Optics Express, vol. 14, no. 17, pp. 7579-7586, 2006.

[11] V. K. Polyanskii, O. V. Angelsky, and P. V. Polyanskii, "Scattering-induced spectral changes as a singular optical effect," Optica Applicata, vol. 32, no. 4, pp. 843-848, 2002.

[12] M. T. Tavassoly, A. Nahal, and Z. Ebadi, "Image formation in rough surfaces," Optics Communications, vol. 238, no. 4-6, pp. 252-260, 2004.

[13] M. Dashtdar and M. T. Tavassoly, "Determination of height distribution on a rough interface by measuring the coherently transmitted or reflected light intensity," Journal of the Optical Society of America A, vol. 25, no. 10, pp. 2509-2517, 2008.

[14] M. Dashtdar and M. T. Tavassoly, "Redshift and blueshift in the spectra of lights coherently and diffusely scattered from random rough interfaces," Journal of the Optical Society of America A, vol. 26, no. 10, pp. 2134-2138, 2009.

[15] M. T. Tavassoly and M. Dashtdar, "Height distribution on a rough plane and specularly diffracted light amplitude are Fourier transform pair," Optics Communications, vol. 281, no. 9, pp. 2397-2405, 2008. 

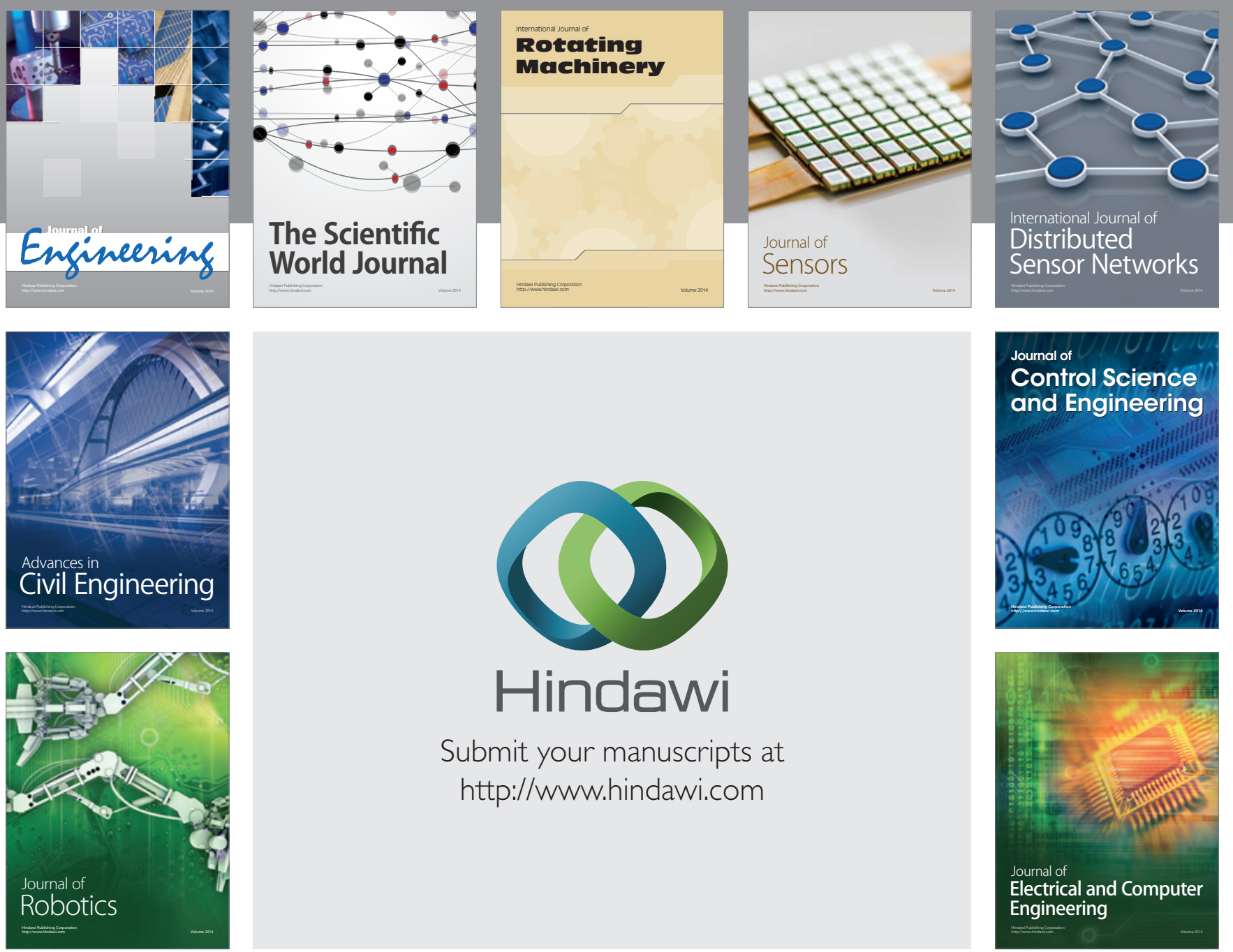

Submit your manuscripts at

http://www.hindawi.com
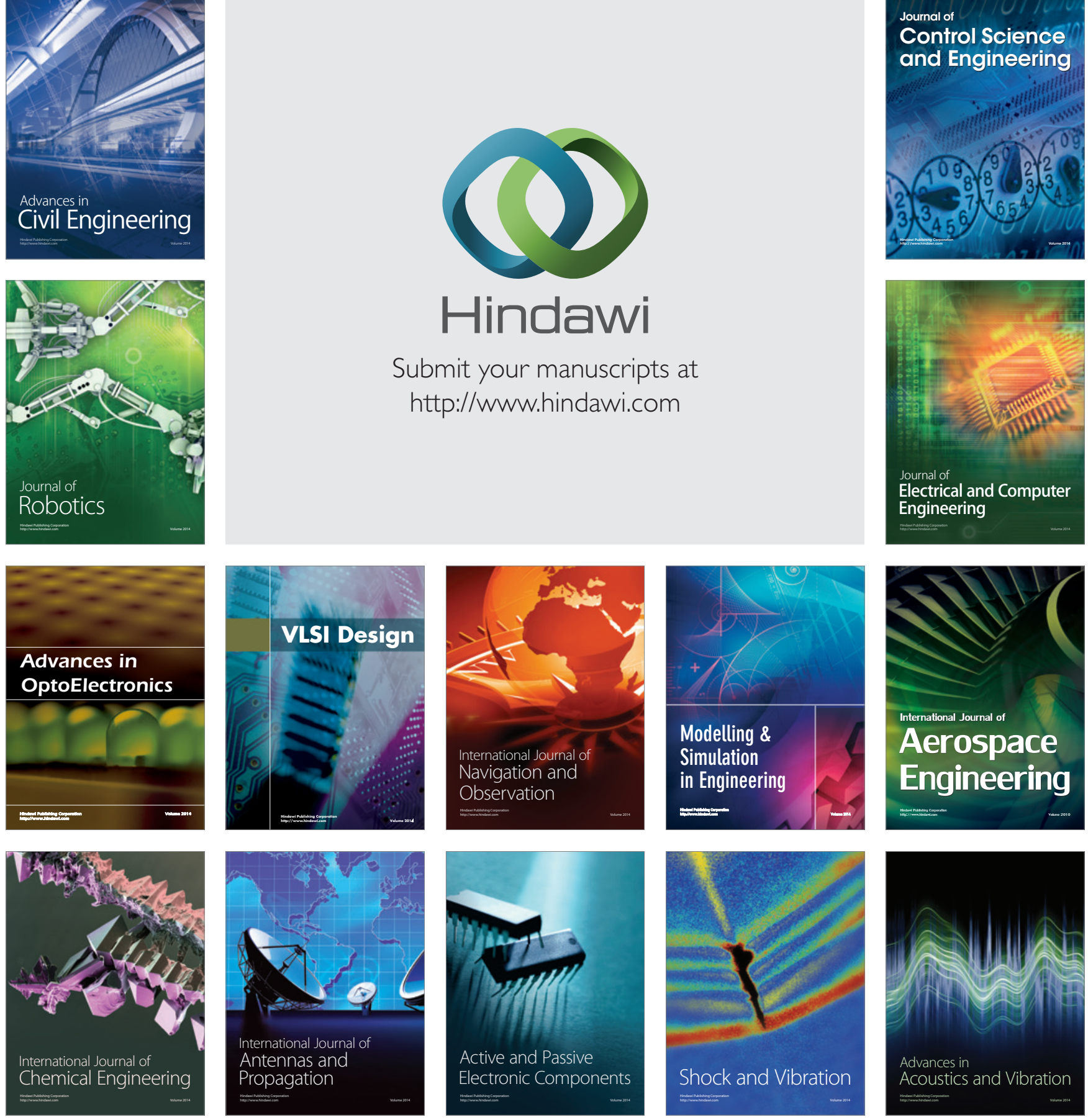